\title{
Miranda
}

Revue pluridisciplinaire du monde anglophone /

Multidisciplinary peer-reviewed journal on the English-

speaking world

$21 \mid 2020$

Modernism and the Obscene

\section{Krakauer-Tagg Duo : du souffle et des marteaux pour abattre les murs du confinement}

\section{Cyril Camus}

\section{OpenEdition}

Journals

Édition électronique

URL : http://journals.openedition.org/miranda/28782

DOI : 10.4000/miranda.28782

ISSN : 2108-6559

Éditeur

Université Toulouse - Jean Jaurès

\section{Référence électronique}

Cyril Camus, « Krakauer-Tagg Duo : du souffle et des marteaux pour abattre les murs du

confinement », Miranda [En ligne], 21 | 2020, mis en ligne le 12 octobre 2020, consulté le 16 février

2021. URL : http://journals.openedition.org/miranda/28782 ; DOI : https://doi.org/10.4000/miranda. 28782

Ce document a été généré automatiquement le 16 février 2021.

\section{(c) $(1)$}

Miranda is licensed under a Creative Commons Attribution-NonCommercial-NoDerivatives 4.0 International License. 


\title{
Krakauer-Tagg Duo : du souffle et des marteaux pour abattre les murs du confinement
}

\author{
Cyril Camus
}

1 C'est le hasard qui a voulu que Breath \& Hammer, le premier album du Krakauer-Tagg Duo, sorte en plein milieu d'une période où une grande part du monde se calfeutrait pour ralentir l'hécatombe provoquée par la COVID-19. Nous naviguions alors entre canapé et salle de bain, avec rares détours réglementés par la supérette ou la pharmacie. Beaucoup balançaient entre angoisse exacerbée par les actualités, deuils impossibles de proches isolés, et télétravail écrasant. Les «essentiels 》 devaient substituer au télétravail des équipées terrifiantes dans des transports en commun, des usines, des supermarchés ou des hôpitaux devenus de potentiels foyers de contagion. Certains n'en pouvaient plus d'être enfermés sans fin avec les mêmes personnes, et rêvaient d'être seuls. D'autres, seuls, enviaient les premiers. La sortie de Breath \& Hammer le 8 mai 2020, dans ce contexte inédit, est, certes, un hasard, mais on n'aurait guère pu imaginer de meilleur moment pour l'apparition de cet album précis. Baume au cœur et décloisonnement ultime, le travail de Krakauer et Tagg, qui a toujours consisté à briser beaucoup de murs symboliques, est un must-listen pour les confinés de partout.

2 Le "souffle» évoqué dans le titre de l'album, c'est celui que David Krakauer insinue dans sa clarinette. Ce sexagénaire new-yorkais est depuis longtemps une légende pour les amateurs de klezmer jovial, de jazz déchaîné et de hip-hop insolite. Il n'a pas manqué de succès dans le domaine de la musique savante, comme le Diapason d'or obtenu en collaboration avec le Kronos Quartet, pour leur interprétation (1997) de Dreams and Prayers of Isaac the Blind (1994) du compositeur argentin Osvaldo Golijov ${ }^{1}$, ou le prix Pulitzer de musique obtenu par l'œuvre de chambre Tempest Fantasy (2004) composée par l'Américain Paul Moravec pour Krakauer et le trio de pianistes Trio Solisti ${ }^{2}$. C'est toutefois en dynamitant les cloisons entre divers genres de musique populaire que Krakauer a semblé s'épanouir le plus, faisant partager à des publics divers son énergie, son goût de l'invention, sa passion pour la musique traditionnelle juive et sa volonté 
d'impliquer cette tradition dans toutes les fusions culturelles imaginables. Entre 1988 et 1996, ces traits se sont exprimés au sein du groupe The Klezmatics, dont le style klezmer $^{3}$ se mêle régulièrement à des sonorités et des rythmes jazz, rock ${ }^{4}$ ou $s k a^{5}$. Il a ensuite créé son propre orchestre Klezmer Madness! et il a invité à y participer le rappeur, pianiste et accordéoniste canadien Joshua Dolgin, dit « Socalled», dont le hiphop éclectique est aussi très marqué par l'influence klezmer'. Ils ont multiplié ensemble les mélanges entre klezmer/jazz à la Krakauer (déjà très métissé7) et hip-hop à la Socalled (donc tout aussi cosmopolite, et tout aussi féru de culture yiddish ${ }^{8}$ ), à l'image de leur

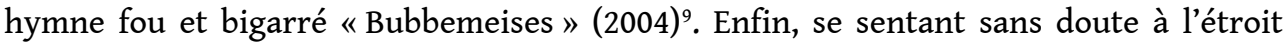
entre des horizons culturels si limités, Krakauer et Socalled ont formé un autre groupe (Abraham Inc.) avec Fred Wesley, célèbre tromboniste afro-américain qui a joué aux côtés de James Brown ou au sein du Count Basie Orchestra et de Parliament-Funkadelic. Ainsi naquirent deux albums rehaussant le cocktail ashkénaze d'une touche de funk: Tweet Tweet en $2012^{10}$ et Together We Stand en $2019^{11}$.

3 Parmi les récentes aventures musicales de Krakauer (en plus de la formation d'un autre groupe appelé Ancestral Groove), il y a le Krakauer-Tagg Duo qu'il forme avec l'autre architecte de Breath \& Hammer. En effet, le «marteau », ou plutôt les marteaux, dont il est question, c'est ceux qui se trouvent dans le piano de Kathleen Tagg. Pianiste sudafricaine de renom, également résidente de New York depuis 2001, elle s'emploie aussi à briser les murs qui séparent les genres musicaux. Son association avec Andre Petersen, autre pianiste sud-africain, sur l'album Where Worlds Collide (2017), en est un exemple notable. L'univers sonore de Tagg, plutôt classique et expérimental, et celui de Petersen, résolument jazz, s'y mêlent dans une course effrénée ${ }^{12}$. Une autre des cocréations de Tagg est le spectacle (et l'album correspondant) Soul of Fire (2014). Avec le violoniste classique Piet de Beer, le bassiste de jazz Charles Lazar, et le percussionniste spécialisé dans la world music Joseph Avergal, Tagg y accompagne la chanteuse soprano Zanne Stapelberg dans son interprétation passionnée et entraînante de douze tangos, flamencos et chansons traditionnelles d'Espagne et d'Amérique latine ${ }^{13}$.

4 Le Krakauer-Tagg Duo a été formé en 2012. Les deux musiciens éponymes ont depuis créé et joué plusieurs spectacles ensemble - notamment The Keepers of the Flame en $2017^{14}$ et Voyages en 2018 (voir Kelly), et l'album Breath \& Hammer, qui donne l'impression d'être une synthèse de ces rencontres créatives, est en fait tiré de leur premier spectacle, également intitulé Breath \& Hammer, et présenté au public pour la première fois le 17 janvier 2016, dans la salle de concert National Sawdust, à Brooklyn, dans le cadre du festival artistique FERUS qu'y organise, tous les ans, la maison de production VisionIntoArt ${ }^{15}$.

5 Sur la page de présentation du spectacle, sur le site du duo, une citation du critique journalistique Bill Milkowski qualifie Kathleen Tagg de pianiste "aventureuse» (Milkowski). Plus loin, le texte de présentation dit qu'elle est une "exploratrice sonore " assimilable à un "savant fou " («'mad-scientist' South African sound explorer »; « About »). En effet - comme Krakauer a su tirer de sa clarinette mille sons étonnants à travers le travail sur les rythmiques propres aux divers styles musicaux qu'il a abordés et mélangés, en repoussant toujours plus loin la tessiture de l'instrument, et en pratiquant la respiration circulaire ${ }^{16}$ - Tagg utilise des techniques de jeu étendues ${ }^{17}$. Ainsi, elle traite volontiers le cadre du clavier comme un instrument de percussion, et elle joue tout aussi volontiers sur un " piano préparé $»^{18}$. Dans le cadre de Breath \& Hammer, elle a poussé ses expérimentations bien plus loin. À travers la « 
préparation » des cordes avec du papier et du tissu, mais aussi en mobilisant cordes et marteaux de maintes façons hétérodoxes (en pinçant les marteaux comme des cordes de harpe, par exemple, ou en frottant les cordes avec une mèche d'archet, comme s'il s'agissait des cordes d'un violon ou d'un violoncelle), et en créant des boucles électroniques à partir de samples des sons qu'elle produit, elle parvient à transformer son piano en orchestre complet, créant tout un environnement sonore riche qui enveloppe les étirements onduleux du son tantôt suave, tantôt strident de la clarinette de Krakauer ${ }^{19}$.

6 L'album Breath \& Hammer pousse cette logique encore plus loin que le spectacle. Comme l'explique le texte de présentation qu'on trouve dans le livret du CD (et sur la plateforme d'écoute et de vente Bandcamp ${ }^{20}$ ), chacune des neuf pistes de l'album a été créée à partir de centaines d'enregistrements de sons tirés par Krakauer de sa clarinette et par Tagg de son piano préparé. En créant des samples de leurs propres interprétations, et en les mêlant pour former neuf morceaux électro-acoustiques composites, chacun étant issu d'environ quarante sources différentes, mais avec toujours les mêmes interprètes, le duo aurait pu aboutir à l'équivalent sonore de la créature de Frankenstein ${ }^{21}$. C'est le contraire qui se produit. Cette suite est bien un assemblage de sons émis à des moments et dans des conditions différentes; elle est aussi un assemblage de styles musicaux hétéroclites, point culminant des carrières de deux musiciens voués à l'hybridité générique ; enfin, Krakauer et Tagg ne se privent pas des ruptures de rythme et de ton qu'on attend d'un artiste à la discographie empreinte de $j a z z^{22}$ et d'une autre qui s'inscrit dans le domaine de la musique expérimentale ${ }^{23}$. Pourtant, les maîtres mots semblent être douceur et harmonie. Si l'écoute de Breath \& Hammer favorise indéniablement l'immersion dans un univers sonore qui captive l'imagination comme ceux que décrit Josh Kun dans Audiotopia (2005) ${ }^{24}$, cet univers est très loin de la violence, des ténèbres glacées et des cris d'angoisse qu'évoquent ceux de certains célèbres compositeurs avant-gardistes, également adeptes des ruptures et des techniques de jeu étendues, tels que Ligeti ou, surtout, Penderecki ${ }^{25}$. La musique expérimentale et composite du Krakauer-Tagg Duo se situe, au contraire, dans un registre apaisant et chaleureux. Krakauer lui-même le soulignait lorsqu'il évoquait des morceaux joués au cours du spectacle Voyages, déjà présents au programme du spectacle Breath \& Hammer, et partiellement repris sur l'album :

Some of the (avant-garde) repertoire is crazy and very violent, but this actually is a very peaceful, calm, meditative sort of piece. It builds quite a bit and gets crazy in the middle, but in a friendly sort of way. (cité dans Kelly)

7 La question qui peut alors se poser est : comment détruit-on des murs sans violence ? Il s'agit bien sûr de favoriser la communication entre les deux côtés d'un mur, pour permettre aux gens (ou aux cultures) de part et d'autre de faire comme si le mur n'existait pas. Créer des espaces musicaux (pour rester dans le champ de la métaphore de Josh Kun) où les cultures se rencontrent à travers le mélange de leurs traditions a été le but avoué d'à peu près tous les groupes et les projets développés par Krakauer ${ }^{26}$. Chez Tagg aussi, les liens culturels et humains sont un thème récurrent, comme on peut le voir notamment à travers le titre de ses premiers albums : Connection (2014) - une suite de pièces du compositeur américain Jake Heggie, que Tagg interprète en duo avec la cantatrice new-yorkaise Regina Zona - et Where Worlds Collide, déjà évoqué, avec Andre Petersen. Breath \& Hammer ne fait pas exception. Le texte de présentation de l'album affirme ainsi : " Fundamentally Breath \& Hammer is about connections between people and a celebration of identity through music ${ }^{27}$. Le spectacle éponyme était 
accompagné d'enregistrements en direct de gros plans de Tagg, de Krakauer et de leurs instruments pendant qu'ils jouaient, projetés sur un écran derrière eux, et, lors de leurs concerts dans la salle Pierre Boulez à Berlin (2019), les deux musiciens étaient entourés d'une sorte de vaste paravent transparent qui faisait office d'écran, et les images mouvantes de leurs propres gestes musicaux les enveloppaient comme d'immenses doubles spectraux, formant une installation conçue par l'artiste multimédia américain Jesse Gilbert ${ }^{28}$, et intitulée The Ties That Bind $U s^{29}$. Puisque les vidéos qui composent The Ties That Bind Us représentent l'acte physique de production de la musique, on peut supposer que les «liens " évoqués dans le titre de l'installation sont la musique ellemême. Quant à " nous» («us»), c'est un déictique qui a l'avantage de pouvoir désigner, alternativement voire simultanément, Tagg et Krakauer, et/ou Tagg et Krakauer et le public, et/ou Tagg et Krakauer et les musiciens du monde entier qui les inspirent et qui sont leurs amis et collaborateurs, et/ou les musiciens du monde entier en général, et/ ou les musiciens du monde entier et leur(s) public(s), et/ou l'humanité en général.

Sur le plan de la thématisation des « liens » et des échanges, l'album a bien sûr pour lui, comme le spectacle, la diversité d'origine des œuvres interprétées. « November 22 » est ainsi une composition de Kinan Azmeh, clarinettiste syrien qui vit à New York et se produit souvent en Allemagne et en Syrie ${ }^{30}$. «Shron » est l'œuvre de Roberto Rodriguez, percussionniste new-yorkais d'origine cubaine, spécialisé dans la fusion du klezmer et des musiques d'Amérique latine ${ }^{31}$. "Demon Chopper» est une création de Rob Curto, accordéoniste philadelphien d'origine italienne, spécialisé dans la musique folk américaine et les musiques brésiliennes, notamment le forró ${ }^{32}$. « Parzial » et « Ebuhuel » sont des compositions du saxophoniste et compositeur avant-gardiste et multigenres John Zorn ${ }^{33}$, initialement créées pour son groupe de klezmer/free jazz Masada, et déjà présentes sur un album de morceaux de Zorn joués par Krakauer, sorti en $2012^{34}$. " Moldavian Journey" est l'œuvre d'Emil Kroitor, célèbre accordéoniste klezmer moldave ${ }^{35}$. Enfin, "The Geyser» est une création commune de Krakauer et Tagg, " Rattlin' Down the Road» est de Krakauer et, surtout, «Berimbau » est une composition de Tagg (également présente sur son album Where Worlds Collide ${ }^{36}$ ), et la pianiste précise que le titre est le nom d'un instrument de musique brésilienne ${ }^{37}$, mais fait aussi référence à un instrument similaire utilisé dans son Afrique du sud natale ${ }^{38}$.

Si l'album n'est pas, contrairement au spectacle, enveloppé dans The Ties That Bind Us, il n'en a pas moins eu ses propres extensions audiovisuelles, et ce, en réaction à la pandémie et au confinement. Ces événements ont en effet conduit à l'annulation de la tournée prévue pour accompagner la sortie de l'album, et celle-ci a donc été remplacée par les Krakauer \& Tagg's Sunday Connections, une émission hebdomadaire en ligne, diffusée en direct sur Youtube, Facebook, et les différents sites de Krakauer et Tagg ${ }^{39}$, produite et animée par les deux artistes, qui s'efforcent, selon le mot de Krakauer dans la deuxième vidéo, d'«apporter de la musique depuis chez eux jusque chez leurs spectateurs", en ces temps "sacrément difficiles $»^{40}$. Les deux musiciens se filment dans leur appartement new-yorkais, et en visioconférence avec leurs amis et collaborateurs, également confinés chez eux, avec, en général, un ou des extraits de concert enregistrés avant la période du confinement, et d'éventuels extraits d'entretiens également réalisés pré-confinement. Pour chaque vidéo, les invités choisissent une œuvre caritative, et les spectateurs qui le peuvent sont invités à faire un don. La première vidéo (29 mars 2020) est un entretien avec Kinan Azmeh autour de sa composition « November 22 », de ce qu'elle signifie pour lui (son explication prend la 
forme d'une méditation sur la notion mouvante de «foyer » pour un expatrié), suivi d'un extrait d'un concert d'Azmeh jouant " November 22 », accompagné de l'orchestre de chambre du festival Morgenland d'Onasbrück, puis d'un extrait du spectacle Breath \& Hammer, où Krakauer et Tagg jouent leur version du morceau d'Azmeh. La deuxième vidéo ( 5 avril 2020) est une présentation d'Abraham Inc., avec des extraits d'une visioconférence avec Fred Wesley, et de nombreux extraits de visioconférences et d'entretiens pré-confinement avec les différents (et nombreux) autres membres du groupe, ainsi que des extraits de concerts, notamment une interprétation, par le groupe, du tube funk de Wesley « House Party » (1980). La troisième vidéo (12 avril) est un entretien en visioconférence avec l'écrivain polonais Krzysztof Czyżewski et son compatriote enseignant et musicien Michał Moniuszko, qui travaillent tous deux au sein de la fondation Borderland et du Borderland Center of Arts, Culture and Nations, à Sejny, deux institutions visant à promouvoir les échanges culturels (notamment à travers la musique klezmer) entre la Pologne, la Lituanie, la Biélorussie, et les autres pays dont la région frontalière de Sejny peut être vue comme le carrefour. La vidéo se termine sur «Human Music» (2017), un extrait de The Keepers of the Flame, spectacle de Tagg et Krakauer organisé avec la collaboration de nombreux musiciens du monde entier, et avec l'aide de la fondation Borderland. La quatrième vidéo (19 avril) est une visioconférence avec Rob Curto, qui explique ce qu'est le forró, parle de la genèse de sa composition «Demon Chopper ", qu'il joue un peu en direct de chez lui, seul avec son accordéon, avant que Kathleen Tagg n'aille au piano pour montrer les différentes étapes qu'elle doit suivre, pour sa reprise de «Demon Chopper» avec Krakauer, pour transformer son piano en orchestre de percussions brésiliennes. Enfin, un extrait du spectacle Breath \& Hammer conclut la vidéo en montrant le "produit fini». Dans la cinquième vidéo ( 26 avril), Tagg et Krakauer parlent, avec le réalisateur américain Eric Steel, de son film Minyan (2020), et de la bande originale qu'ils ont composé pour le film, avec Krakauer à la clarinette et Tagg au piano et au violoncelle ${ }^{41}$. D'autres visioconférences du même genre étaient prévues, avec Jesse Gilbert le 3 mai, Andre Petersen le 17 mai, Socalled le 24 mai, et enfin, le 31 mai, avec Jerome Harris, bassiste et guitariste d'Ancestral Groove, et Sarah MK, chanteuse qui s'est produite ponctuellement avec Abraham Inc. ${ }^{42}$

Il semble cependant que la sortie de leur album le 8 mai ait débordé les deux producteurs-animateurs de web-émission en herbe, puisqu'après l'entretien avec Eric Steel, on ne trouve plus que trois courtes vidéos sur la playlist des Sunday Connections sur Youtube, et aucune ne contient d'invité ou d'extraits d'entretiens ou de concerts préconfinement. Au lieu de cela, Krakauer et Tagg interprètent, en direct de l'appartement, un morceau par vidéo : d'abord, le standard klezmer "Der Gasn Nign ", ensuite une composition de Krakauer intitulée "Offering Nigun» (dont le seul enregistrement qui existait jusque-là était une piste de l'album Krakauer Live in Krakow, enregistré avec Klezmer Madness! et Socalled au club Indingo de Cracovie en juin 2003, et édité en CD en 2004), et, pour finir, «Moldavian Journey» d'Emil Kroitor. Dans la première (celle du 3 mai), Krakauer explique que lui et Tagg vont faire deux concerts, d'une heure et demie chacun, en direct de l'appartement, le dimanche suivant (événement qui a effectivement eu lieu le 10 mai), et que ça demande donc trop de préparation pour pouvoir aussi tourner un épisode normal de Sunday Connections. On peut supposer que l'emploi du temps postérieur à la sortie de l'album n'a pas permis de reprendre l'émission comme prévu ${ }^{43}$. 
11 Pendant la période du confinement (ou les différentes périodes de confinement, selon les pays), il y a eu beaucoup de musiciens s'essayant à la diffusion de concerts virtuels dans des salles dédiées ${ }^{44}$ ou depuis chez eux ${ }^{45}$, ou à des sortes de "bœufs Zoom ", où plusieurs musiciens jouent ensemble en visioconférence ${ }^{46}$. Il y a même eu un certain nombre d'artistes célèbres dans d'autres domaines que la musique décidant de chanter un peu ou de jouer un air face à leur webcam ${ }^{47}$. Les quelques chercheurs qui ont commencé à analyser ces phénomènes s'accordent pour voir les mêmes notions de construction de lien social et de solidarité mises en avant dans chacune de ces interactions entre ces personnalités et les millions d'internautes qui suivent leurs publications en ligne, ou qui sont prévenus de ces récitals virtuels par les articles participant ensuite à leur viralité. Ainsi, Anita Datta a beau conclure un bref article sur ces «chorales virtuelles» en niant catégoriquement leur capacité à créer une expérience partagée comparable à un concert « en présentiel », elle n'en commence pas moins sa démonstration avec le constat suivant:

Early into lockdown audio-visual content began surfacing on social media, presenting 'virtual' ensemble performances created by layering individually recorded videos of musicians performing in isolation. Such multi-track videos present a spirit of communitas and fortitude, invoking the power of music to 'bring people together'. (Datta 1)

Selon Datta, la réalité empirique contredirait cette profession de foi en raison d'appareils d'enregistrement inadéquats (dans le cas notamment des smartphones et autres webcams), et des difficultés à synchroniser et accorder en direct plusieurs voix et/ou plusieurs instruments enregistrés à des endroits différents, dans des conditions différentes, et avec des appareils de trop mauvaise qualité pour bien s'entendre mutuellement. Elle s'appuie sur l'expérience, selon elle « universellement » décevante et stressante, des musiciens qui s'y sont essayés ${ }^{48}$. Des sources aussi vagues paraissent cependant difficiles à vérifier. Le champ d'expérience auquel elle s'intéresse est par ailleurs très restreint. En effet, si le début de son article décrivait le phénomène de façon assez large, en se référant aux " musiciens professionnels ", elle ne parle en fin de compte que des chorales de chant classique et religieux, et elle n'évoque que l'expérience des membres de ces chorales, pas celle des spectateurs.

Roberta Lamb et Robbie MacKay adoptent une perspective plus large, s'intéressant de façon globale à la manière dont les échanges musicaux se sont accrus entre personnes confinées, non seulement à travers les vidéos en ligne de célébrités et de musiciens, mais aussi avec les chants sur les balcons et autres initiatives spontanées de diverses populations (voir Lamb et MacKay). Ils n'évoquent pas les difficultés et limitations techniques posées par l'enregistrement et la diffusion sur l'Internet. Ils s'appuient par contre, non sur des témoignages « universels", mais sur des articles de pédagogues de la musique, d'animateurs sociaux et d'ethnomusicologues (avec des liens hypertextes renvoyant aux articles en question). Ils passent aussi une partie de leur démonstration à analyser la tonalité et le rythme des morceaux de musique folk joués par l'acteur américain Steve Martin, dans son jardin, sur les vidéos de banjo qu'il a mises en ligne fin mars 2020. Enfin, ils s'intéressent aussi, pour cet exemple, à la façon dont Martin utilise sa persona publique ainsi que la mise en scène de sa vidéo pour amplifier l'impression de familiarité, de camaraderie, qu'inspire sa courte intrusion dans la navigation quotidienne, émaillée d'actualités terrifiantes, des internautes confinés. Leurs conclusions sont sans appel: quelles que soient les conditions, les échanges musicaux sont un medium fondamental pour tisser des liens de solidarité et bâtir le 
sentiment d'une communauté partagée, et les phénomènes d'accroissement des échanges musicaux en ligne ou entre balcons pendant le confinement ont été un exemple expressif de ce pouvoir d'apaisement et de rapprochement de la musique.

Dans un environnement comme celui-là, le travail de Krakauer et Tagg semble avoir donné naissance à l'un des univers créatifs les plus adaptés au besoin collectif de compenser symboliquement la séparation physique radicale et généralisée que décrit la formule « distanciation sociale ». Détruire les murs et les cloisonnements (entre genres musicaux, entre cultures, entre communautés), construire des liens, des ponts, mélanger, métisser, brasser, c'est l'obsession que traduisent les productions musicales de chacun des deux musiciens, et Breath \& Hammer ne témoigne assurément pas d'un quelconque essoufflement en la matière. Que l'aspiration se traduise toujours par une réussite ou non ${ }^{49}$ importe peu : son expression reste systématique, et évidente dans la moindre pratique des deux musiciens, en duo ou avec d'autres. Non seulement l'album, sa fusion de genres musicaux, et son intense et chaleureuse expressivité, reflètent cette obsession et cette expérience dans la recherche de ponts et de liens entre les gens et les cultures, mais son caractère composite et expérimental préfigurait la réussite dans le domaine de la mise en ligne de vidéos confinées. En effet, Krakauer et Tagg ont produit un album dont les dialogues entre instruments résonnent avec beaucoup de naturel, alors même qu'il s'agit en fait d'un agrégat de centaines de samples, et que les nombreux instruments qu'on croit entendre sont en fait les mille emplois bizarres que Tagg a trouvés à faire de son piano. Après cet exploit, il ne semble, rétrospectivement, pas si difficile de produire en catastrophe des vidéos elles-mêmes composites (car mélangeant visioconférences en direct, concerts à la maison, extraits d'anciens entretiens ou concerts), et de parvenir à en faire le modèle de convivialité et de chaleur humaine requis en ces temps troublés. Tout concourt à faire des Sunday Connections exactement ce modèle : des regards pétillants des deux producteurs-animateurs lorsqu'ils parlent de musique avec leurs invités/collaborateurs/amis au contenu à la fois serein et passionné de leurs échanges, en passant par la simplicité douillette du décor. Cette série de vidéos, malheureusement incomplète, aura finalement étendu et enrichi avec beaucoup d'à-propos ce qui a été le projet multimédia d'expression et de création de convivialité de Breath \& Hammer de sa naissance sur la scène de National Sawdust en 2016 à son apparition sur les plateformes de vente et d'écoute en 2020, du décloisonnement des genres et des cultures au déconfinement des esprits.

\section{BIBLIOGRAPHIE}

\section{MUSICOLOGIE ET ETUDES CULTURELLES}

Balderston, Daniel, Mike Gonzalez et Ana M. López (eds.). Encyclopedia of Contemporary Latin American and Caribbean Cultures. Abingdon/New York : Routledge, 2000.

Barzel, Tamar. New York Noise: Radical Jewish Music and the Downtown Scene. Bloomington/ Indianapolis : Indiana University Press, 2015. 
Cuthbert, Michael Scott. «Free Improvisation: John Zorn and the Construction of Jewish Identity through Music». Harvard Judaica Collection, Student Research Papers, $\mathrm{n}^{\circ} 7$ (2001) : 1-31.

Datta, Anita. « 'Virtual Choirs' and the Simulation of Live Performance under Lockdown». Social Anthropology (2020) : 1-2. Wiley Online Library. 20 mai 2020. Consulté le 9 juin 2020. <https://onlinelibrary.wiley.com/doi/full/10.1111/1469-8676.12862>.

Glaser, Amelia. « The Idea of Yiddish. Re-globalizing North American Jewish Culture». In The Routledge Handbook of Contemporary Jewish Cultures. Ed. Laurence Roth et Nadia Valman. Abingdon/ New York : Routledge, 2015, 2017. 259-271.

Goldberg, Randall. « David Krakauer from Klezmer Madness! to Abraham Inc.: A Topical Narrative of Musical Identities». Musica Judaica, Vol. 21, 5776 (2015-2016) : 65-112.

Harnsberger, Lindsey. Essential Dictionary of Music. Los Angeles : Alfred Publishing, 1997. Jones, Barrie (ed.). The Hutchinson Concise Dictionary of Music. 1999. New York/Londres : Routledge, 2014.

Kun, Josh. Audiotopia: Music, Race and America. Berkeley/Los Angeles/Londres : University of California Press, 2005.

Lamb, Roberta et Robbie MacKay. « Steve Martin's Banjo and Other Music Played From Coronavirus Isolation Show How the Arts Connect Us». The Conversation. 31 mars 2020. Consulté le 10 juin 2020.

$<\mathrm{https}$ ///theconversation.com/steve-martins-banjo-and-other-music-played-from-coronavirusisolation-show-how-the-arts-connect-us-134519>.

Mitchell, Helen R. « Fear and the Musical Avant-Garde in Games: Interviews with Jason Graves, Garry Schyman, Paul Gorman and Michael Kamper ». Horror Studies, Vol. 5, n 1 (2014) : 127-144.

Nyman, Michael. « Towards (a Definition of) Experimental Music». 1974. In Michael Nyman: Collected Writings. Ed. Pwyll ap Siôn. 2013. Londres/New York : Routledge, 2016. 177-202.

Thomsett, Michael C. Musical Terms, Symbols and Theory. An Illustrated Dictionary. Jefferson : McFarland \& Company, Inc., 1989.

MAGAZINES, JOURNAUX ET SITES D'ARTISTES

« About ». KrakauerTaggDuo.com. Consulté le 3 juin 2020. <https://www.krakauertaggduo.com/about.html>.

« David Krakauer \& Kathleen Tagg Launch New Weekly Livestream Series: Sunday Connections with Krakauer \& Tagg». 21C Media Group. 15 avril 2020. Consulté le 9 juin 2020.

$<$ https://21cmediagroup.com/2020/04/15/david-krakauer-kathleen-tagg-launch-new-weeklylivestream-series-sunday-connections-with-krakauer-tagg/>.

Gilbert, Jesse. Site officiel. Page d'accueil. Consulté le 7 juin 2020.

$<$ http://jessegilbert.net/>.

Hughes, William. « Let Steve Martin Soothe Your Soul through the Most Relaxing of Mediums: Banjo Music». The A.V. Club. 21 mars 2020. Consulté le 9 juin 2020.

$<$ https://news.avclub.com/let-steve-martin-soothe-your-soul-through-the-mostrela-1842440593>.

Kelly, Justin. « David Krakauer and Kathleen Tagg Present 'Treasure Trove of Sounds' in Recital ». The Chautauquan Daily. 8 juillet 2018. Consulté le 2 juin 2020.

$<$ https://chqdaily.com/2018/07/david-krakauer-and-kathleen-tagg-present-treasure-trove-ofsounds-in-recital/>. 
Knopper, Steve. « Kinan Azmeh: Syrian Clarinetist Stranded in Beirut Thanks to Trump Ban». Rolling Stone. $1^{\text {er }}$ février 2017. Consulté le 8 juin 2020.

<https://www.rollingstone.com/music/music-features/kinan-azmeh-syrian-clarinetiststranded-in-beirut-thanks-to-trump-ban-117856/>

Makowski, Jenna. « The Eclectic Sounds of Abraham Inc. ». DC Life 5 (2012) : 40-43.

Milkowski, Bill, « 'World Of Krakauer' Concert Fuses Jazz, Funk, Klezmer ». Downbeat. 16 février 2016. Consulté le 3 juin 2020.

<https://downbeat.com/news/detail/world-of-krakauer-concert-fuses-jazz-funk-klezmer>.

Solomon, Jon. « David Krakauer on the Origins and the Name of Abraham Inc. ». Westword.

11 novembre 2011. Consulté le 7 juin 2020.

$<$ https://www.westword.com/arts/david-krakauer-on-the-origins-and-the-name-of-abrahaminc-5788501>.

\section{SOURCES VIDEO ET AUDIO EN LIGNE}

« 07. Rock the Belz-Theodore Bikel \& Socalled». 19 juillet 2015. Consulté le 4 juin 2020. <https://www.youtube.com/watch?v=4NVX2PeLwGQ>.

3 Daft Monkeys. «Perfect Stranger Lockdown Cyber-Busk». 2 juin 2020. Consulté le 9 juin 2020. $<$ https://www.facebook.com/3daftmonkeys/videos/762528870951805/>.

Abraham Inc., featuring David Krakauer, Fred Wesley and Socalled. Together We Stand. 15 octobre 2019. Consulté le 28 mai 2020.

<https://abrahaminc.bandcamp.com/album/together-we-stand>.

Abraham Inc. Tweet Tweet. 25 septembre 2012. Consulté le 28 mai 2020. <https://abrahaminc.bandcamp.com/album/tweet-tweet>.

«Berimbau-Kathleen Tagg ». 20 octobre 2017. Consulté le 8 juin 2020.

<https://taggpetersenduo.bandcamp.com/track/berimbau-kathleen-tagg>.

«Breath and Hammer Acoustic | Virtual Concert». 7 juin 2020. Consulté le 8 juin 2020. $<$ https://www.youtube.com/watch?time_continue=2097\&v=TpRGbOQM-x0>.

« Breath \& Hammer: The Ties That Bind Us. David Krakauer \& Kathleen Tagg ». 25 mars 2020. Consulté le 7 juin 2020.

< https://www.youtube.com/watch?v=YTgZDhTGsAc>.

« Brearth [sic] \& Hammer: An Immersive Fusion of Sound and Images». Consulté le 7 juin 2020. <https://boulezsaal.de/event/david-krakauer-kathleen-tagg/2019_03_09_1900>.

«Clarinet Virtuoso David Krakauer and Pianist Kathleen Tagg Share an Intimate Performance of Their Original Work and Selected Pieces From Home in Our Latest Music Mondays Online

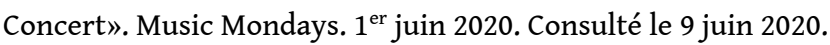

<https://www.facebook.com/145851504032/videos/578004199793014/>.

« David Krakauer-Bubbemeises (feat. Socalled \& Klezmer Madness) ». 9 juin 2015. Consulté le 28 mai 2020.

<https://www.youtube.com/watch?v=xE9DZvloVmM>.

« Dropkick Murphys-Streaming Up From Boston». 17 mars 2020. Consulté le 9 juin 2020.

<https://www.youtube.com/watch?v=5j06g0TE_Ao>.

«FKZ | Emil Kroitor \& The Trance-Moldavian Express | Stranik \& Husan ». 24 septembre 2019.

Consulté le 8 juin 2020.

$<$ https://www.youtube.com/watch?v=0w3JlpwRpzM>. 
«FKZ : Krakauer \& Kronos Quartet ». 18 mars 2020. Consulté le 27 mai 2020.

<https://www.youtube.com/watch?v=p_sJLY-5qTU>.

« Hot 8 Brass Band-Love Will Tear Us Apart (Jazz Foundation of America Emergency Fund Concert) ». 5 juin 2020. Consulté le 9 juin 2020.

<https://www.youtube.com/watch?v=RYtTLDxY_0g>.

« John Zorn/Roberto Rodriguez - Aguares, Book of Angels Vol. 23 ». 4 novembre 2016. Consulté le 8 juin 2020.

<https://www.youtube.com/watch?v=VlTqNwb0veg>.

« Kathleen Tagg \& Andre Petersen play 'Rapela' by Moses Molelekwa » 10 octobre 2018. Consulté le 3 juin 2020.

<https://www.youtube.com/watch?v=0R-sKNWKSyo>.

« Kathleen Tagg's 'Piano Orchestra'. Breath \& Hammer w David Krakauer. Nov 22 by Kinan Azmeh arr. Tagg ». 29 mars 2020. Consulté le 3 juin 2020.

<https://www.youtube.com/watch?v=SBJLaBWX4UE>.

« Keepers of the Flame ». KrakauerTaggDuo.com. Consulté le 2 juin 2020.

<https://www.krakauertaggduo.com/keepers-of-the-flame.html>.

«Klezkalypso (Teach the Ska)». 6 novembre 2014. Consulté le 28 mai 2020.

$<$ https://www.youtube.com/watch?v=WYS90DrkMto>.

« Krakauer \& Tagg's Sunday Connections \#2 with Fred Wesley and Abraham Inc. ». 17 avril 2020.

Consulté le 9 juin 2020.

$<$ https://www.youtube.com/watch?v=h8rz3uKgakw\&list=PLCeqXTnHPUrqxOpqI7zMAX4iWTlsf61d>.

Krakauer, David et Kathleen Tagg. Breath \& Hammer. 8 mai 2020. Consulté le 5 juin 2020. <http://krakauertagg.bandcamp.com/album/breath-hammer>.

Krakauer, David. Pruflas: The Book of Angels, Vol. 18. 27 mars 2012. Consulté le 8 juin 2020. $<$ https://boomkat.com/products/pruflas-the-book-of-angels-vol-18>.

« Listen: David Krakauer and Kathleen Tagg Present 'Breath and Hammer' ». WQXR. 28 juillet 2016. Consulté le 2 juin 2020.

<https://www.wqxr.org/story/listen-breath-and-hammer-david-krakauer-and-kathleen-tagg/>.

Matuto. Brazilian Carnaval in the Appalachian Mountains. Consulté le 8 juin 2020. <https:// matutomusic.com/media/audio/>.

Playlist « Krakauer \& Tagg Sunday Connections Broadcasts From Home ». Dernière mise à jour le 30 mai 2020. Consulté le 9 juin 2020.

<https://www.youtube.com/playlist?list=PLCeqXTnHPUrqxOpqI7zMA-X4iWTlsf61d>.

Playlist « Moravec: Tempest Fantasy / Mood Swings / B.A.S.S. Variations ». 25 mars 2020. Consulté le 27 mai 2020.

<https://www.youtube.com/playlist?list=OLAK5uy_l8uAcNc0rj1E3_Sgglk4yXiRwyhtr8rrA>.

Playlist « Socalled-Ghettoblaster (Full Album) ». 10 janvier 2016. Consulté le 28 mai 2020. <https://www.youtube.com/playlist?list=PLhrglt2nmIGiRluuRVR97aoDKvJx0zWM7>.

Socalled. « UNLVD » . 3 mai 2011. Consulté le 4 juin 2020.

<https://socalledmtl.bandcamp.com/track/unlvd>

Stapelberg, Zanne et Kathleen Tagg. Soul of Fire. 14 avril 2014. Consulté le 31 mai 2020. $<$ https://daddykool.com/UPC/6009704960164>. 
Tagg, Kathleen and Andre Petersen. Where Worlds Collide. 20 octobre 2017. Consulté le 31 mai 2020. <https://kathleentagg.bandcamp.com/album/where-worlds-collide>.

« The Klezmatics-Elijah Rock ». $1^{\text {er }}$ février 2016. Consulté le 28 mai 2020.

<https://www.youtube.com/watch?v=cJkr23u1Usg>.

The Klezmatics. Tuml = leben. 17 octobre 2008. Consulté le 16 octobre 2020. <https://theklezmatics.bandcamp.com/album/tuml-leben>.

« Together at Home with First Aid Kit». 23 mars 2020. Consulté le 9 juin 2020.

<https://www.youtube.com/watch?v=eSKJl19GRw0\&t=63s>.

AUTRES

Shelley, Mary. Frankenstein; or The Modern Prometheus. 1818. In Four Gothic Novels: The Castle of

Otranto, Vathek, The Monk, Frankenstein. Oxford/New York : Oxford University Press, 1994. 461-606.

\section{NOTES}

1. Voir « FKZ: Krakauer \& Kronos Quartet».

2. Voir Playlist « Moravec: Tempest Fantasy / Mood Swings / B.A.S.S. Variations ».

3. Voir «The Klezmatics, Tuml = leben »

4. Voir « The Klezmatics - Elijah Rock».

5. Voir « Klezkalypso (Teach the Ska)».

6. Voir Playlist « Socalled-Ghettoblaster (Full Album)».

7. «Krakauer and [Michael] Alpert immersed themselves in the study of Greek, Turkish and Balkan music at the same time they were learning klezmer. In fact, Krakauer notes that after he began to perform with klezmer groups, it was his ability to see connections between various musical styles that helped him find a voice in Jewish music » (Goldberg 88).

8. "The Canadian Jewish rapper DJ Socalled opens one of his songs with a soliloquy by the Yiddish singer Theodor Bikel: [...] one of many [samples] that DJ Socalled [...] uses in fusion music that integrates Yiddish, English and French, among other languages.» (Glaser 266). Outre le rappeur québécois d'origine congolaise Kamenga Mbikay, dit "Sans Pression », qui intervient en français sur la chanson « (Rock the) Belz» (2007) évoquée par Glaser (voir « 07. Rock the Belz Theodore Bikel \& Socalled»), Socalled a aussi enrôlé Enrico Macias parmi les intervenants de sa chanson « UNLVD» (2011) (voir Socalled, « UNLVD»).

9. Voir « David Krakauer - Bubbemeises (feat. Socalled \& Klezmer Madness)».

10. Voir Abraham Inc., Tweet Tweet.

11. Voir Abraham Inc., featuring David Krakauer, Fred Wesley and Socalled. Together We Stand.

12. Voir Tagg et Petersen. Where Worlds Collide.

13. Voir Stapelberg et Tagg. Soul of Fire.

14. Voir « Keepers of the Flame».

15. Voir « Listen: David Krakauer and Kathleen Tagg Present 'Breath and Hammer' ».

16. "Circular breathing [is a] breathing technique used by wind instrument players where air is inhaled through the nose at the same time air is expelled from the mouth. This technique allows for sound to be sustained for an indefinite length of time » (Harnsberger 31).

17. «[The phrase] extended technique [refers,] in general, [to] nontraditional methods of obtaining sounds from musical instruments. » (Jones 203).

18. «[A] prepared piano [is] a piano whose strings have been altered in some way. Either pieces of metal, wood, felt, or other objects have been added to mute or alter sound quality » (Thomsett 
174). Pour un exemple connu d'emploi de pianos préparés par Tagg (et Andre Petersen), voir « Kathleen Tagg \& Andre Petersen play 'Rapela' by Moses Molelekwa ».

19. Selon les termes du texte de présentation déjà cité : « Krakauer's use of extended techniques, improvisation, and circular breathing on the clarinet, along with Tagg's prowess inside the piano to remake it as a harp, a zither, a drum, and a cello creates a collective sound that completely transforms these two acoustic instruments » (voir « About »).

On peut voir un aperçu du travail de Tagg sur la vidéo « Kathleen Tagg's 'Piano Orchestra'. Breath \& Hammer w David Krakauer. Nov 22 by Kinan Azmeh arr. Tagg", où plusieurs images superposées se concentrent chacune sur une des boucles musicales lancées par la pianiste au cours de l'interprétation du morceau « November $22 »$.

20. Voir Krakauer et Tagg. Breath \& Hammer.

21. Celle-ci est, en effet, également une création composite, patchwork de divers morceaux de cadavres, que le protagoniste du roman de Shelley a assemblés avant de leur donner vie, mais on sait que Frankenstein se montre pour le moins déçu du résultat esthétique de son travail d'assemblage: "no mortal could support the horror of that countenance. A mummy again endued with animation could not be so hideous as that wretch. I had gazed on him while unfinished; he was ugly then; but when those muscles and joints were rendered capable of motion, it became a thing such as even Dante could not have conceived " (Shelley 491).

22. «Krakauer's engagement with klezmer blends musical, cultural, and personal motivations, particularly in relation to his experiences playing jazz. In a 2005 interview, he claimed that in the 1970s he suffered a 'crisis in confidence' regarding jazz: 'I was afraid that I had nothing to say. What could I do after [John] Coltrane, Coleman Hawkins, Charlie Parker, Sidney Bechet, [and] Louis Armstrong? [...] And ultimately, I started playing with some of those [klezmer] musicians. So suddenly my jazz background and listening to all these amazing Turkish and Greek and Albanian players came together. I felt like I had found a musical home.' » (Goldberg 73, 88).

23. «Experimental composers have [...] brought about the alteration of timbre by inserting objects between the strings [...]. Cage devised the prepared piano as a one-man percussion band and Steve Reich describes his Phase Patterns as 'literally drumming on the keyboard.' Alternatively, auxiliary objects may be placed between the keyboard and the performer who activates them to produce sounds, as in Kosugi's Distance" (Nyman 192). On a vu dans les précédents paragraphes à quel point le travail de Kathleen Tagg s'inscrit dans cette tradition.

24. "[S]ongs can be understood as audiotopias. [...] [M] usic functions like a possible utopia for the listener, that [...] is experienced not only as sound that goes into our ears and vibrates through our bones but as a space that we can enter into, encounter, move around in, inhabit, be safe in, learn from [...]. [...] [M]usic and songs are [...] almost-places of cultural encounter that may not be physical places but nevertheless exist in their own auditory somewhere. [...] I can put on a song and live it, hear it, get inside its notes and chords, get inside its narratives and follow its journeys and paths » (Kun 2-3).

25. C'est ce qui conduit Helen Mitchell à conclure, à la fin de la reproduction d'une série d'entretiens qu'elle a menés avec des compositeurs de musique de jeux vidéo d'horreur : «In the depiction of fear and horror, the 'otherness' of avant-garde music continues to be a source of inspiration to composers [...] involved in films and games. Associations between the musical avant-garde and what might be termed 'the dark side' are firmly entrenched within popular culture [...]. Although avant-garde music might not be 'pulling the crowds' in terms of concert hall box office receipts, within the popular context of horror and visual media it has become the sound of fear » (Mitchell 142-143). Le Hongrois György Ligeti est bien sûr cité en référence au cours de ces entretiens (139) et le nom du Polonais Krzysztof Penderecki y apparait à de multiples reprises $(133,139,140,141)$, notamment (133) pour l'utilisation abondante que Stanley Kubrick a fait de ses compositions dans la bande originale de son film The Shining (1980). On peut ajouter à cet exemple le fait que le Lontano (1967) de Ligeti figurait aussi dans la bande originale de ce film, 
et que cinq morceaux de Penderecki avaient déjà été utilisés par William Friedkin pour celle de The Exorcist (1973). Même pour des films qui ne sont pas tout à fait des films d'horreur mais qui s'y apparentent ou jouent tout au moins sur des moments d'extrême tension dramatique, ces deux compositeurs semblent s'imposer. On retrouve ainsi le Lontano de Ligeti dans Shutter Island (2010) de Martin Scorsese, thriller policier balançant entre récit de détection et horreur psychologique, son Requiem (1963-1965) dans Godzilla (2014) de Gareth Edwards, remake américain apocalyptique du kaiju eiga éponyme (1954) d'Ishirô Honda, et le Thrène pour les victimes d'Hiroshima (1959-1961) de Penderecki apparaît dans la scène de guérilla urbaine du film de science-fiction dystopique Children of Men (2006) d'Alfonso Cuarón, et à plusieurs reprises dans la troisième saison (2017) de la série Twin Peaks (1990-2017) de David Lynch.

26. Voilà par exemple comment le projet culturel du groupe Abraham Inc. a été décrit, par Fred Wesley (dans un entretien de 2012 avec Jenna Makowski pour le magazine DC Life) : «Abraham is the father of us all. We all come from the seed of Abraham. Black, white, Jewish, Christian, Muslim-it all comes from one seed, and it's all incorporated into one new music » - et par Krakauer (dans un entretien avec Jon Solomon pour le site du journal Westword) : "It's totally logical in terms of the direction music is going in general now. And just totally logical politically like in the world today when you can bring cultures together. Not just a collage, because that's not really interesting, but a real dialogue between cultures » (cités dans Goldberg 103 ; voir Makowski 43 pour la publication initiale des propos de Wesley; voir Solomon pour ceux de Krakauer).

27. Voir Krakauer et Tagg, Breath \& Hammer.

28. Voir Gilbert, site officiel.

29. On en voit un aperçu dans « Breath \& Hammer: The Ties That Bind Us. David Krakauer \& Kathleen Tagg». Un peu plus d'explications et d'extraits photographiques, audio et vidéo peuvent être trouvés sur la page «Brearth [sic] \& Hammer: An Immersive Fusion of Sound and Images ".

30. C'est lui qui, sur son trajet retour vers New York, début 2017, a été bloqué quelque temps à Beyrouth à cause du décret de Donald Trump interdisant l'accès au territoire américain aux ressortissants de l'Iran, de l'Irak, de la Libye, de la Somalie, du Soudan, de la Syrie et du Yémen (voir Knopper).

31. On peut écouter un album complet de compositions klezmer de John Zorn arrangées par Rodriguez de façon à les habiller de sonorités et de rythmes typiques des musiques cubaines, à la page « John Zorn/Roberto Rodriguez-Aguares, Book of Angels Vol. 23 ».

32. «Brazilian music/dance style from the Northeast, traditionally played in trio with accordion, triangle and marching drum. Forro can also include other instruments but [...] remains accordion-led» (Balderston, Gonzalez et López 590). On peut écouter des productions du groupe de «bluegrass brésilien » Matuto, dirigé par Curto, à la page Matuto. Brazilian Carnaval in the Appalachian Mountains.

33. Pour plus d'éléments sur Zorn, le mouvement de musique avant-gardiste, rock et jazz qu'il a fondé autour du klezmer et de l'identité juive, et les liens entre lui et Krakauer, voir Cuthbert, et voir aussi Barzel.

34. Un aperçu de chaque piste, un peu plus long que sur d'autres sites de vente, peut être entendu à la page Krakauer, Pruflas.

35. Voir « FKZ | Emil Kroitor \& The Trance-Moldavian Express | Stranik \& Husan ».

36. Voir « Berimbau-Kathleen Tagg».

37. «A pitched percussion instrument of bantu African origin, [...] found mainly in the Brazilian state of Bahia, and [...] used to accompany capoeira. A wooden bow with a metal or rubber string, whose tension is varied by means of a coin or wedge at one end, it produces a limited range of oscillating tones. The string is struck rhythmically with a thin wooden stick, and the sound 
amplified by means of a gourd resonator attached to the lower end of the bow » (Balderston, Gonzalez et López 166).

38. Elle explique cela à environ 32'10" après le début de la vidéo "Breath and Hammer Acoustic Virtual Concert ».

39. Playlist « Krakauer \& Tagg Sunday Connections Broadcasts From Home ».

40. "This is really a heck of a time for everybody, so, hope you're doing okay, and, we decided to do this series to bring music from our home to yours.» Ma traduction. "Krakauer \& Tagg's Sunday Connections \#2 with Fred Wesley and Abraham Inc. ».

41. Précision : il s'agit d'un vrai violoncelle, pas d'un piano transformé en violoncelle. La vidéo contient un montage d'enregistrements des deux musiciens en train de jouer l'une des parties de la bande originale.

42. Voir « David Krakauer \& Kathleen Tagg Launch New Weekly Livestream Series: Sunday Connections with Krakauer \& Tagg ».

43. Le duo a en effet repris les concerts promotionnels. Certains sont effectués chez eux (voir "Clarinet Virtuoso David Krakauer and Pianist Kathleen Tagg Share an Intimate Performance »). D'autres sont effectués dans des salles dédiées, mais le public reste quand même chez lui, le concert étant malgré tout enregistré et retransmis en ligne. C'est le cas de «Breath and Hammer Acoustic | Virtual Concert », enregistré dans l'église méthodiste de Saranac pour l'association The Hill and Hollow Music et le média Mountain Lake PBS.

44. Exemple : « Dropkick Murphys-Streaming Up From Boston ».

45. Exemple : « Together at Home with First Aid Kit ».

46. Exemples : 3 Daft Monkeys. «Perfect Stranger Lockdown Cyber-Busk » et « Hot 8 Brass BandLove Will Tear Us Apart (Jazz Foundation of America Emergency Fund Concert) ».

47. Voir Hughes pour deux exemples en un article.

48. "Singers of all standards have universally reported the experience to be stressful, disorienting and musically dissatisfying " (Datta 1).

49. Randall Goldberg note ainsi, à propos d'Abraham Inc. : "[T]he musicians consistently emphasize their collaboration as a paradigm of Black-Jewish relations. For example, while promoting a performance at a Jewish music festival in Maryland, Krakauer told the Washington Jewish Week: 'What's been very beautiful about this project is that it feels like an ongoing discussion between Jews and African-Americans, a beautiful cultural exchange.' It is difficult to find such press in African-American periodicals for the band, and maybe that tells us something about how it is perceived by Black America. Of course, a harmonious musical relationship is not the same as a nationwide Black-Jewish coalition, and Wesley may have noted the limitations of Abraham Inc.'s metaphor in an interview aired by American Public Media: 'Blacks and Jews both like to eat, dance, and sing. That's very important. And shaking booty is important to both cultures, and we will come together on that if nothing else.' If the band does not reflect the reality of Black-Jewish relations, however, they at least provide an 'audiotopia' where the ensemble and their fans can feel good about this fraught relationship » (Goldberg 104-105). 
INDEX

Thèmes : Music, Dance

Keywords : klezmer, fusion, jazz, experimental music, electro-acoustic music, video, Internet Mots-clés : klezmer, fusion, jazz, musique expérimentale, musique électro-acoustique, vidéo, Internet

\section{AUTEURS}

\section{CYRIL CAMUS}

Professeur agrégé en CPGE / Docteur

Lycée Ozenne

cyril.camus@hotmail.fr 